Arroyo, M., Belytschko, T., Finite crystal elasticity of carbon nantubes based on the exponential Cauchy-Born rule, Physical Review B, Vol. 69, pp. 115415-0, 2004

\title{
Finite crystal elasticity of carbon nanotubes based on the exponential Cauchy-Born rule
}

\author{
M. Arroyo* and T. Belytschko \\ Department of Mechanical Engineering, Northwestern University, Evanston, Illinois 60208, USA
}

\begin{abstract}
A finite deformation continuum theory is derived from interatomic potentials for the analysis of the mechanics of carbon nanotubes. This nonlinear elastic theory is based on an extension of the Cauchy-Born rule called the exponential Cauchy-Born rule. The continuum object replacing the graphene sheet is a surface without thickness. The method systematically addresses both the characterization of the small strain elasticity of nanotubes and the simulation at large strains. Elastic moduli are explicitly expressed in terms of the functional form of the interatomic potential. The expression for the flexural stiffness of graphene sheets, which cannot be obtained from standard crystal elasticity, is derived. We also show that simulations with the continuum model combined with the finite element method agree very well with zero temperature atomistic calculations involving severe deformations.
\end{abstract}

\section{INTRODUCTION}

Despite the brittle fracture observed in experiments ${ }^{1}$ and simulations, ${ }^{2,3}$ and the development of plasticity predicted by calculations,,${ }^{4,5}$ carbon nanotubes can sustain very large elastic deformations without developing lattice defects, and atomistic calculations have shown that indeed the nonlinear mechanical response of these nanostructures very closely resembles that of macroscopic buckled elastic shells. ${ }^{6} \mathrm{Re}-$ cently, continuum mechanics concepts have often been applied to study the mechanics of carbon nanotubes. The resilience of carbon nanotubes to severe mechanical deformation has been experimentally demonstrated ${ }^{7-12}$ which suggests that elastic models are applicable over a large range of deformations. However, consistent finite deformation continuum theories for nanotubes have only recently been investigated. $^{13}$

The characterization of the small strain response of carbon nanotubes through elastic moduli such as Young's modulus, Poisson's ratio, or the flexural rigidity is now ubiquitous in the literature. ${ }^{14-18}$ The extraction of the elastic moduli from an atomistic model, such as analytical potentials, ${ }^{19-21}$ tight-binding models, ${ }^{22}$ or $a b$ initio calculations, ${ }^{16,17}$ is commonly performed by post-processing data from numerical computations through approximate fits to polynomials or finite difference approximations of derivatives. ${ }^{17,23}$ This procedure has some limitations. For instance, the error in $a b$ initio elastic properties calculated from the second derivative of the strain energy with respect to the axial strain can be as high as $10 \%$ due to the numerical approximation of the derivatives. ${ }^{16}$ Furthermore, these approaches do not provide detailed information about the relation between the moduli and the atomistic potential.

Continuum theories have also been applied to the analysis of various aspects of the mechanics of carbon nanotubes (CNTs) such as the vibrational properties, ${ }^{12}$ the energetics of the transverse deformation, ${ }^{9,24}$ and the buckling behavior by linearized stability analysis. ${ }^{625}$ The most common approach is to idealize carbon nanotubes as cylindrical linearly elastic thin shells, thereby ignoring all nonlinearities. The definition of a wall thickness is required for such a model. This thick- ness has often been assumed to be the graphite interlayer distance $t=0.34 \mathrm{~nm}$. Given a thickness, and a value for Young's modulus and Poisson's ratio, thin shell theory provides a formula for the bending modulus of the wall. It was noticed $^{6}$ that the predictions of this theory with $t=0.34 \mathrm{~nm}$ lead to elastic moduli inconsistent with the actual properties of CNTs, extracted for instance from atomistic calculations. This problem has been formally overcome either by selecting the precise thickness for which the thin shell formulas are consistent (around $t=0.066 \mathrm{~nm}),{ }^{6}$ or by defining the effective thickness in bending as an independent elastic parameter. ${ }^{18}$ Both ad hoc approaches manifest the inadequacy of thin shell theory, which is aimed at bulk materials forming structures very thin in one dimension, for an intrinsically two-dimensional material such as the graphene monolayer. In particular, the mechanism that endows graphene sheets with bending stiffness does not result from a thickness across which part of the material is in tension and part in compression, as will become clear later. It has been noted by some authors ${ }^{22}$ that the two-dimensional (2D) nature of graphene sheets, which are crystalline films one atom thick, renders the notion of a wall thickness rather awkward from a purely mechanistic point of view. Following this idea, it is now common to express Young's modulus in units of surface tension. 17,26

In this paper, the method for bridging the atomistic description of curved single layer crystalline films and continuum mechanics theories put forward in Ref. 13 is combined with bond-order potentials. ${ }^{27-29}$ This continuum theory is based on an extension of the Cauchy-Born rule called the exponential Cauchy-Born rule, which accounts for the curvature of the film. Analytical results on the elastic in-plane and bending properties of graphene as well as numerical simulations of carbon nanotubes are presented. Unlike conventional linear elastic thin shell analysis, the present nonlinear theory acknowledges the two-dimensional nature of graphene (the continuum object replacing the crystalline monolayer is a $2 \mathrm{D}$ surface without thickness), and is rigorously derived from an underlying atomistic potential.

Section II outlines the limitations of existing crystal elasticity theories for carbon nanotubes, as well as the theoretical 
foundation of the present theory. Section III describes the formulation of the continuum constitutive response in terms of the interatomic potential. The precise definitions of the infinitesimal elastic moduli of carbon nanotubes, and their explicit expression in terms of the functional form of the interatomic potential, are provided in Sec. IV. It is shown that these analytically derived moduli coincide with those extracted from atomistic calculations. In Sec. V, the simulation method developed by combining the continuum mechanics surface model and the finite element method is shown to accurately mimic the energetics, the large deformation morphologies, and the structural instabilities of the parent atomistic model in the fully nonlinear regime. In a companion paper ${ }^{30}$ the mechanics of thick multiwalled carbon nanotubes containing millions of atoms are explored.

\section{FINITE CRYSTAL ELASTICITY FOR CURVED MONOLAYERS}

The relation between the elastic moduli of bulk crystalline materials and the interatomic interactions is well established in modern molecular theories of elasticity. There are two equivalent approaches for extracting the elastic moduli from the atomistic description, ${ }^{31,32}$ namely the method of the homogeneous deformations, ${ }^{33-35}$ also called the Cauchy-Born hypothesis, ${ }^{36}$ and the method of the long waves within lattice-dynamical theories. ${ }^{31}$ For examples of the application of these classical methods to the in-plane response of graphene, see Refs. 37 and 38, respectively. The method based on the Cauchy-Born rule is purely static but, unlike the asymptotic long wave limit of lattice dynamics, describes the mechanics of crystals at finite strains. This method gives rise to continuum constitutive models systematically derived from the atomistic model in hand. Furthermore, the derived constitutive models inherit the symmetries of the underlying lattice. Finite crystal elasticity has been used, for instance, to obtain elastic moduli and study the stability of strained crystals. ${ }^{33-35,39,40}$ Recently, these ideas have been cast in a computational framework to solve general boundary value problems with complex geometries or loading, in what is called the quasicontinuum method. ${ }^{41}$ This method can handle defects and fracture, by adaptively refining the continuum description down to the atomic level where required. A simpler version, the so-called local quasicontinuum method, combines the finite element method and finite crystal elastic constitutive relations; successful applications include the simulation of nanoindentation in silicon based on an analytical potential and the tight binding method, ${ }^{42,43}$ and an analysis of the polarization switching of ferroelectric single crystals based on an ab initio Hamiltonian. ${ }^{44}$

Given the crystalline nature of carbon nanotubes, and the large elastic (reversible) deformations they exhibit, finite crystal elasticity appears to be appropriate for their mechanical analysis. As recently suggested, ${ }^{13}$ the standard theories aimed at space-filling crystals do not capture the effects of the curvature of crystalline monolayers deforming in three dimensions such as nanotubes. The general idea behind standard finite crystal elasticity in the case of space-filling crystals is sketched below, its limitations for carbon nanotubes illustrated, and the extended theory briefly outlined.

Space-filling continuum bodies can be mathematically represented by subsets of the ambient Euclidean space. Consider the finite deformation of this body. Let $\Phi$ be the deformation that maps the undeformed body $\Omega_{0} \subset \mathbb{R}^{3}$, into $\mathbb{R}^{3}$. If $X$ denotes a point in the undeformed body, its image after deformation is $x=\Phi(X)$. The deformed body is $\Omega$ $=\Phi\left(\Omega_{0}\right)$, and is also a subset of $\mathbb{R}^{3}$. The deformation gradient is the derivative of $\Phi, \mathbf{F}=D \Phi=\partial \Phi / \partial X \in \mathbb{R}^{3 \times 3}$. At each point $X$, the deformation gradient is a linear transformation from $\mathbb{R}^{3}$ into $\mathbb{R}^{3}$, which locally characterizes the deformation, and maps "infinitesimal" material vectors from the undeformed into the deformed body, $d \mathbf{x}=\mathbf{F} d \mathbf{X}$. ${ }^{45}$

The central hypothesis behind molecular theories of elasticity at finite strains is that, at the scale of the atomic spacing, the deformation of the crystal is homogeneous. Consequently, as the crystalline solid deforms, lattice vectors undergo a linear transformation. ${ }^{32,33,46}$ This approach is often abstracted through the Cauchy-Born rule: ${ }^{36,40}$

$$
\mathbf{a}=\mathbf{F A},
$$

where $\mathbf{A}$ denotes an undeformed lattice vector and $\mathbf{a}$ the same vector in the deformed crystal. This rule links the atomistic and the continuum deformations. Complex lattices, that is lattices with more than one atom in the unit cell, require a special treatment as detailed in the following section. The Cauchy-Born rule is restricted to regions where the crystal is free of defects, slips, and other inhomogeneities or special crystallographic phenomena. Its validity and range of applicability has recently been rigorously studied by methods of nonlinear analysis. ${ }^{47}$

Now consider a crystalline monolayer such as a graphene sheet deforming arbitrarily in three dimensions. It is natural in this case to treat the continuum solid as a surface, a curved two-dimensional body without thickness. Indeed, the twodimensional nature of the lattice does not suggest any meaningful thickness; owing to the Born-Oppenheimer approximation, the binding energy depends exclusively on the positions of this two-dimensional arrangement of atoms. It is postulated that the atoms lie on the surface, and therefore the lattice vectors are chords of the surface. ${ }^{13}$

The appropriate framework to describe two-dimensional continua deforming in three dimensional Euclidean space is continuum mechanics on manifolds. ${ }^{48}$ The undeformed body $\Omega_{0}$, which represents the planar ground configuration of graphene, is now a subset of $\mathbb{R}^{2}$. It is mapped by the deformation into the deformed body $\Omega$, a surface in $\mathbb{R}^{3}$. In this context, the deformation gradient $\mathbf{F}$ is called the tangent of the configuration $T \Phi$, and it maps infinitesimal material vectors of the undeformed body $\Omega_{0}$ into vectors which are tangent to the surface $\Omega$ (see Fig. 1).

The standard Cauchy-Born hypothesis $\mathbf{a}=\mathbf{F A}$ produces tangent vectors instead of chords, i.e., $\mathbf{F A} \in T_{x} \Omega$, where $T_{x} \Omega$ denotes the tangent linear space to the surface $\Omega$ at $x$, and therefore does not capture the effect of curvature. A generalized kinematic hypothesis based on the differential geometry concept of the exponential map has been proposed in Ref. 13. The fundamental idea is to compose the standard 


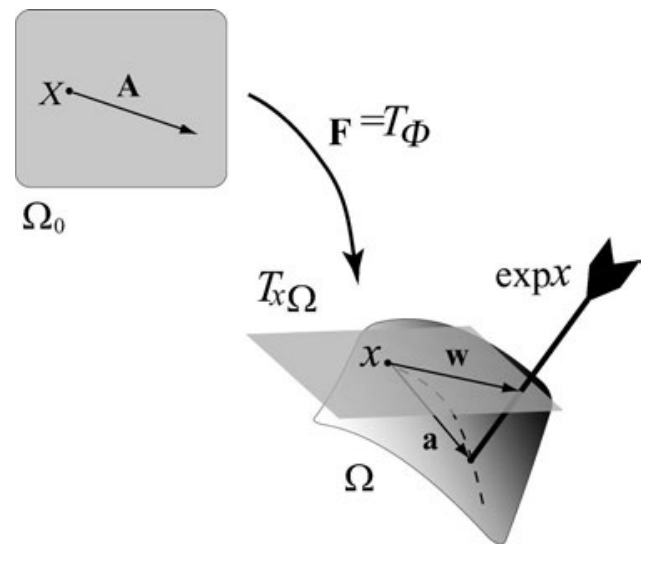

FIG. 1. Illustration of the surface kinematics and the exponential Cauchy-Born rule. The exponential map transforms the vector $\mathbf{w}$ $=$ FA tangent to the surface into a chord of the surface $\mathbf{a}$.

Cauchy-Born rule with the exponential map, ${ }^{49}$ which naturally maps the tangent space onto the curved surface. This is accomplished by the so-called exponential Cauchy-Born rule

$$
\mathbf{a}=\exp \circ \mathbf{F A}
$$

where exp denotes the exponential map of $\Omega$ at the point from which a emanates. The exponential map "brings" the tangent vector $\mathbf{w}=\mathbf{F A}$ to the curved surface, thereby producing a chord (see Fig. 1 for an illustration). More physical insight on this extended hypothesis can be gained by analyzing the simplest case of an atomic chain deforming in two dimensions. $^{50}$

The evaluation of the exponential map requires the knowledge of the geodesic curves of the surface, which in general entails the integration of a system of two differential equations in which the Christoffel symbols are the coefficients. Thus the map described by Eq. (2) is nonlocal and difficult to evaluate exactly. For this reason, the exponential map is approximated. This results in a local, simple model, in which the deformed geometry of the lattice vectors is expressed in terms of the local deformation of the surface, that is the first and the second fundamental forms. ${ }^{49}$ Note the analogy with standard differential geometry of surfaces, by which the undeformed body $\Omega_{0}$ takes the role of the parametric space, and the deformation map $\Phi$ is the parametrization of the surface $\Omega$. The first and second fundamental forms can be "pulled back" 48 by $\Phi$ to the undeformed body, thereby defining the standard left Cauchy-Green deformation tensor $\mathbf{C}$, and a Lagrangian (extrinsic) curvature tensor $\mathcal{K}$. Similarly, in the context of differential geometry of surfaces, the first and the second fundamental forms are sometimes expressed in the referential coordinates. ${ }^{51}$ By formulating the theory in terms of Lagrangian (material) strain measures, the principle of material-frame indifference is automatically satisfied.

The final result of the extended theory is that a new set of continuum strain measures which represent the deformed geometry of the atomic bonds can be defined in terms of the local deformation of the surface $\Omega$, i.e., in terms of $\mathbf{C}$ and $\mathcal{K}$ (see Appendix A). For instance, the deformed bond length $a$

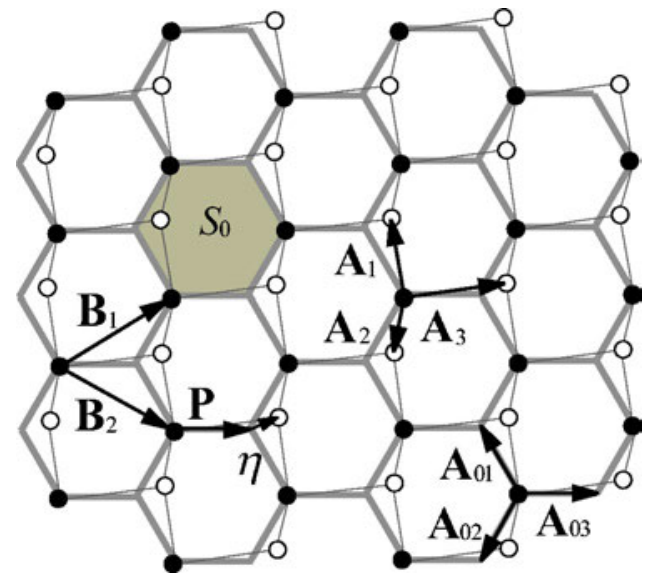

FIG. 2. Graphene honeycomb multilattice and illustration of the inner displacements: the two simple Bravais lattices, depicted in black and white, are relatively shifted by $\boldsymbol{\eta}$, also affecting the bond vectors which are transformed from $\mathbf{A}_{0 i}$ into $\mathbf{A}_{i}$. The unit cell of area $S_{0}$, the Bravais basis vectors $\mathbf{B}_{1}$ and $\mathbf{B}_{2}$, and the shift vector $\mathbf{P}$ are also shown.

of a lattice vector $\mathbf{A}$ after deformation is explicitly written as $a=f(\mathbf{C}, \mathcal{K} ; \mathbf{A})$. Similarly, a continuous variable representing the angle between two lattice vectors $\mathbf{A}$ and $\mathbf{B}$ in the deformed configuration can be written as $\theta=g(\mathbf{C}, \mathcal{K} ; \mathbf{A}, \mathbf{B})$. These derived strain measures are adequate to formulate continuum constitutive functions in terms of bond-order potentials, which only depend on bond lengths and angles. Similar expressions can be derived for other geometric quantities such as dihedral angles.

\section{CONSTITUTIVE LAW FOR GRAPHENE}

One can distinguish three inequivalent bond vectors in the unit cell of the honeycomb lattice of graphene $\mathbf{A}_{0 i}, i$ $=1,2,3$. These bond vectors form three inequivalent angles, labeled such that $\theta_{i}$ is the angle between bond $j$ and bond $k$, and $\{i, j, k\}$ is an even permutation of $\{1,2,3\}$. At the ground state of graphene, the length of each of these bond vectors is denoted by $A_{0}$, and $\theta_{i}=2 \pi / 3, i=1,2,3$. When dealing with graphene, special attention must be paid to the fact that it is a Bravais multilattice; it can be viewed as two woven simple lattices (see Fig. 2). The standard crystal elasticity treatment of multilattices is to assume that the homogeneous deformation affects each of the simple lattices. Additional kinematic variables describing the relative shifts of the simple lattices must be introduced to properly describe the configurations of uniformly strained multilattices. These relative shifts are called inner displacements. ${ }^{33-35,42,46}$ The optical modes are the analog of the inner displacements in lattice dynamical theories. ${ }^{32}$

Let $\boldsymbol{\eta}$ denote the inner displacements field, which following Ref. 42, is defined in the undeformed body, previous to the "macroscopic" deformation $\Phi$. Thus, the lattice vectors are

$$
\mathbf{A}_{i}=\mathbf{A}_{0 i}+\boldsymbol{\eta}, \quad i=1,2,3 .
$$


For some materials, the inner displacements may describe homogeneous phase transformations. ${ }^{42,44}$

Since the configuration of the undeformed lattice depends on $\boldsymbol{\eta}$ [see Eq. (3)], the deformed geometry of the bond vectors does as well; for instance, the lengths of the deformed bond vectors can be written as

$$
a_{i}=\bar{f}\left(\mathbf{C}, \mathcal{K}, \boldsymbol{\eta} ; \mathbf{A}_{0 i}\right), \quad i=1,2,3,
$$

and the three angles these inequivalent bonds form after deformation as

$$
\theta_{i}=\bar{g}\left(\mathbf{C}, \mathcal{K}, \boldsymbol{\eta} ; \mathbf{A}_{0 j}, \mathbf{A}_{0 k}\right), \quad i=1,2,3,
$$

where $\{i, j, k\}$ is an even permutation of $\{1,2,3\}$. The explicit expressions for $\bar{f}$ and $\bar{g}$ follow from Appendix A and Eq. (3).

This finite elasticity theory for curved crystalline monolayers can in principle be combined with any atomistic model. We assume in the following potentials that fall within the bond-order formalism, ${ }^{27}$ and consider the bond-order potentials for hydrocarbons developed by Brenner ${ }^{28}$ which have been widely applied to study the mechanics of carbon nanotubes ${ }^{6,19,52}$ including the nucleation of defects. ${ }^{53,54}$ In these potentials, the energy is expressed in terms of bond lengths and angles as a sum over the bonds:

$$
E=\sum_{i} \sum_{j>i}\left[V_{R}\left(r_{i j}\right)-\bar{B} V_{A}\left(r_{i j}\right)\right]
$$

where the bond-order $\bar{B}$ models the many-body coupling between bond $i j$ and its local environment. It depends on the lengths of the bonds and angles adjacent to the $i j$ th bond. By considering a unit cell, which contains one of each inequivalent bond, and has an undeformed surface area of $S_{0}$ $=(3 \sqrt{3} / 2) A_{0}^{2}$ (see Fig. 2), and using Eqs. (4) and (5), the stored strain energy density (energy per unit undeformed area) of the continuum surface can be written as

$$
\begin{aligned}
W(\mathbf{C}, \mathcal{K}, \boldsymbol{\eta}) & =\frac{1}{S_{0}} E_{\text {cell }}\left(a_{1}, a_{2}, a_{3}, \theta_{1}, \theta_{2}, \theta_{3}\right) \\
& =\frac{1}{S_{0}} \sum_{i=1}^{3}\left[V_{R}\left(a_{i}\right)-\bar{B}\left(a_{j}, a_{k}, \theta_{j}, \theta_{k}\right) V_{A}\left(a_{i}\right)\right]
\end{aligned}
$$

This hyperelastic potential depends on the left Cauchy-Green deformation tensor $\mathbf{C}$ and Lagrangian curvature tensor $\mathcal{K}$ of the surface, and on the inner displacement field $\boldsymbol{\eta}$. The dependence of the energy on the undeformed lattice vectors has been omitted.

The inner displacements can be eliminated at the constitutive level. ${ }^{13,42}$ At each point, given the local deformation, the strain energy density can be minimized with respect to $\boldsymbol{\eta}$ :

$$
\hat{\boldsymbol{\eta}}(\mathbf{C}, \mathcal{K})=\arg \left[\min _{\boldsymbol{\eta}} W(\mathbf{C}, \mathcal{K}, \boldsymbol{\eta})\right]
$$

After this inner relaxation, the effective strain energy density can be written as a function of $\mathbf{C}$ and $\mathcal{K}$ only:

$$
\hat{W}(\mathbf{C}, \mathcal{K})=W[\mathbf{C}, \mathcal{K}, \hat{\boldsymbol{\eta}}(\mathbf{C}, \mathcal{K})]
$$

While a closed-form expression for the hyperelastic potential $W$ in terms of the atomistic potential is available [see Eq. (7)], the evaluation of $\hat{W}(\mathbf{C}, \mathcal{K})$ requires the solution of a bivariate minimization problem. If the graphene sheet is planar, i.e., $\mathcal{K}=\mathbf{0}$, this theory results exactly in standard finite crystal elasticity. The total internal energy functional for the continuum surface is obtained from the surface integral of the hyperelastic strain energy density over the undeformed body:

$$
\Pi_{\mathrm{int}}[\Phi]=\int_{\Omega_{0}} \hat{W}[\mathbf{C}(\Phi), \mathcal{K}(\Phi)] d \Omega_{0} .
$$

Stress measures work conjugate to the strain measures for the surface can be derived from the hyperelastic potential

$$
\mathbf{S}=2 \frac{\partial \hat{W}}{\partial \mathbf{C}}=\left.2 \frac{\partial W}{\partial \mathbf{C}}\right|_{\boldsymbol{\eta}=\hat{\boldsymbol{\eta}}}, \quad \mathbf{m}=\frac{\partial \hat{W}}{\partial \mathcal{K}}=\left.\frac{\partial W}{\partial \mathcal{K}}\right|_{\boldsymbol{\eta}=\hat{\boldsymbol{\eta}}}
$$

It is possible to replace the derivatives of the effective potential $\hat{W}$ by derivatives of the analytically available potential $W$ as long as the inner displacements are in internal equilibrium, as argued in Refs. 42 and 13. The first of these stress measures is an in-plane stress, and corresponds to the second Piola-Kirchhoff stress. ${ }^{48}$ It has units of force divided by length (surface tension), while the second is a momentlike stress that has units of force. These unusual units for stress tensors follow from the fact that the continuum surface has no thickness.

\section{ELASTIC MODULI}

Effective (i.e., at the relaxed inner displacements) Lagrangian elasticity tensors can be obtained by taking second derivatives of the elastic potential with respect to the strain measures:

$$
\mathrm{C}_{\mathrm{a}}=4 \frac{\partial^{2} \hat{W}}{\partial \mathbf{C}^{2}}, \quad \mathrm{C}_{\mathrm{b}}=\frac{\partial^{2} \hat{W}}{\partial \mathcal{K}^{2}}, \quad \mathrm{C}_{\mathrm{c}}=2 \frac{\partial^{2} \hat{W}}{\partial \mathbf{C} \partial \mathcal{K}}
$$

The first of these elasticity tensors is a measure of the inplane stiffness of the surface and is measured in units of force divided by length. It corresponds to the second elasticity tensor. ${ }^{48}$ The second represents the bending stiffness and the third is an in-plane/bending coupling stiffness. Again, the unusual surface tension units for the in-plane elastic modulus are a consequence of the two-dimensional nature of a graphene sheet.

In the calculation of these elastic moduli, even when the inner displacements are in internal equilibrium we cannot replace $\hat{W}$ by $W$, as in Eq. (11); extra terms arise with crossed derivatives of $W$ with respect to the strain measures and the inner displacements. ${ }^{42}$ The evaluation of these moduli at an arbitrary deformation requires $\hat{\boldsymbol{\eta}}(\mathbf{C}, \mathcal{K})$, which in general must be obtained numerically. 


\section{A. In-plane moduli}

We now develop closed-form expressions for infinitesimal elastic moduli in the analytically tractable situation of planar graphene in its ground state, for which the inner displacements vanish. Although considering the small strain elasticity of planar graphene as representative of that of nanotubes may seem a crude approximation, these moduli are available in a closed form, and are found to provide good estimates. Indeed, $a b$ initio calculations show that the elastic moduli of nanotubes differ only slightly from those of planar graphene. ${ }^{16,17,26}$

The in-plane moduli of graphene can be treated following standard methods of crystal elasticity; other results have been recently reported. ${ }^{37}$ Here, the precise expressions in terms of the functional form of the potential are provided, as well as a discussion of how to interpret them. The proposed theory also furnishes expressions for the flexural stiffness. For concreteness, potentials based on the bond-order formalism are considered, although explicit expressions can be obtained for other interatomic potentials.

By methods analogous to those in Ref. 42, the in-plane second elasticity tensor in Eq. (12) can be computed at the relaxed inner displacements as

$$
\mathrm{C}_{\mathrm{a}}=4\left[\frac{\partial^{2} W}{\partial \mathbf{C}^{2}}-\frac{\partial^{2} W}{\partial \mathbf{C} \partial \boldsymbol{\eta}} \cdot\left(\frac{\partial^{2} W}{\partial \boldsymbol{\eta}^{2}}\right)^{-1} \cdot \frac{\partial^{2} W}{\partial \boldsymbol{\eta} \partial \mathbf{C}}\right]
$$

This tensor, evaluated at the ground state of graphene, corresponds with the usual small deformation tensor of elastic moduli.

For moderate deformations, the bond-order function $\bar{B}$ of bond $i j$ in Brenner's potential depends only on the angles adjacent to this bond. ${ }^{20,28}$ In the following, all expressions are evaluated at the equilibrium configuration of graphene, characterized by equal bond angles $2 \pi / 3$, and equilibrium bond length $A_{0}$. Let us denote by $V_{R}^{\prime}$ and $V_{R}^{\prime \prime}$ the first and second derivatives of $V_{R}$ with respect to their only argument, and similarly for $V_{A}$. Let $\bar{B}^{\prime}$ denote the first derivative of the bond order $\bar{B}$ with respect to any of its arguments (when evaluated for graphene at equilibrium, the choice of argument does not matter), while $\bar{B}^{\prime \prime}$ denotes the second derivative of $\bar{B}$ with respect to one of its arguments. By $\bar{B}^{\prime}$,' we denote the second derivative of $\bar{B}$ with respect to the first and the second arguments. Let us define

$$
\begin{gathered}
C_{r r}=V_{R}^{\prime \prime}-\bar{B} V_{A}^{\prime \prime}, \quad C_{\theta \theta}=\frac{3 V_{A}}{A_{0}^{2}}\left(2 \bar{B}^{\prime \prime}-\bar{B}^{\prime}{ }^{\prime}{ }^{\prime}\right), \\
C_{r \theta}=\frac{2 \sqrt{3}}{A_{0}} V_{A}^{\prime} \bar{B}^{\prime} .
\end{gathered}
$$

Lengthy but otherwise straightforward calculations lead to the following expression for the Euclidean components of the in-plane infinitesimal elasticity tensor of graphene:

$$
\mathrm{C}_{i j k l}=\mu\left(\delta_{i k} \delta_{j l}+\delta_{i l} \delta_{j k}\right)+\lambda \quad \delta_{i j} \delta_{k l},
$$

where

$$
\mu=\frac{1}{4 \sqrt{3}}\left[C_{r r}-C_{r \theta}-C_{\theta \theta}-\frac{\left(C_{r r}+C_{\theta \theta}\right)^{2}}{\underline{C_{r r}+C_{r \theta}-C_{\theta \theta}}}\right]
$$

and

$$
\lambda=\frac{1}{4 \sqrt{3}}\left[C_{r r}+C_{r \theta}+C_{\theta \theta}+\frac{\left(C_{r r}+C_{\theta \theta}\right)^{2}}{C_{r r}+C_{r \theta}-C_{\theta \theta}}\right]
$$

are the Lamé coefficients. The underlined terms correspond to the effect of the inner displacements, i.e. the second term on the right hand side of Eq. (13).

The tensor of infinitesimal moduli in Eq. (15) has the general form of a fourth order isotropic tensor. Unlike for bulk materials, this tensor is defined in a two-dimensional space [the indices in Eq. (15) run from 1 to 2]. Thus, the infinitesimal elasticity tensor is isotropic, a well known fact about honeycomb lattices. This does not imply that graphene is isotropic for finite deformations, i.e. its invariance group does not coincide with the group of proper finite rotations $S O(2) .{ }^{48}$ Finite deformations introduce anisotropy in the lattice; in particular the finite deformation required to roll a graphene sheet into a nanotube induces anisotropy observable in the slight variations of the elastic moduli with radius and chirality. ${ }^{55}$

The conventional expressions relating the Lamé coefficients with Young's modulus $Y$ and Poisson's ratio $\nu$ for bulk isotropic linearly elastic materials are

$$
Y=\frac{\mu(3 \lambda+2 \mu)}{\lambda+\mu} \quad \text { and } \quad \nu=\frac{\lambda}{2(\lambda+\mu)} .
$$

Young's modulus and Poisson's ratio are defined from the thought experiment of applying uniaxial tension $\sigma_{11}$ to a prismatic homogeneous isotropic linear elastic body, and measuring the strains in each direction. Then $Y:=\sigma_{11} / \varepsilon_{11}$ and $\nu:=-\varepsilon_{22} / \varepsilon_{11}=-\varepsilon_{33} / \varepsilon_{11}$. One may be tempted to use the expressions in Eq. (18) for graphene. However, it is important to bear in mind that they are defined for bulk materials, and the present theory regards graphene sheets as genuinely two-dimensional objects, for which the standard "plane-strain" or "plane-stress" conditions do not make sense. If one subjects a rectangular planar slab of graphene to uniaxial tension $\sigma_{11}$, measures the strains $\varepsilon_{11}$ and $\varepsilon_{22}\left(\varepsilon_{33}\right.$ is not defined), and adopts the natural definitions $Y_{s}:=\sigma_{11} / \varepsilon_{11}$ and $\nu_{s}:=-\varepsilon_{22} / \varepsilon_{11}$, the resulting expressions in terms of the Lamé coefficients in Eq. (15) are

$$
Y_{s}=\frac{4 \mu(\lambda+\mu)}{\lambda+2 \mu} \quad \text { and } \quad \nu_{s}=\frac{\lambda}{\lambda+2 \mu} \text {, }
$$

where the subscripts emphasize the fact that they refer to a surface continuum. As usual, $\nu_{s}$ is nondimensional. In this case, $Y_{s}$ has units of surface tension, agreeing with the point of view of other authors. ${ }^{26}$ The in-plane shear modulus $G_{s}$ is also expressed in units of surface tension, and coincides with $\mu$. 


\section{B. Bending modulus}

We consider an initially planar graphene sheet. We then calculate the second derivative of the strain energy density with respect to the curvature in a given direction. We are interested in the scalar modulus

$$
\mathrm{C}_{\mathrm{b}}=\frac{\partial^{2} \hat{W}}{\partial \kappa^{2}}
$$

where $\kappa$ is the only nonvanishing principal curvature of the monolayer. Appendix $\mathrm{B}$ describes the derivation of this modulus in terms of the functional form of the interatomic potential. Several intermediate results deserve special attention.

On the one hand, the fact that the second term of Eq. (B4) vanishes reveals that, unlike the in-plane moduli, the infinitesimal bending modulus is insensitive to the inner displacements. On the other hand, it follows from the derivation of Appendix B that for any two-dimensional honeycomb lattice whose interatomic potential depends only on bond lengths and angles (and not on dihedral angles for instance), the infinitesimal bending modulus around the planar state can be written as

$$
\mathrm{C}_{\mathrm{b}}=\sum_{i=1}^{3} \frac{\partial W}{\partial a_{i}} \frac{\partial^{2} a_{i}}{\partial \kappa^{2}}+\sum_{j=1}^{3} \frac{\partial W}{\partial \theta_{j}} \frac{\partial^{2} \theta_{j}}{\partial \kappa^{2}} .
$$

Remarkably, this modulus does not depend on second derivatives of the atomistic potential function. This means that if we naively adopt a quadratic two-body/three-body expansion of the energy of graphene around its ground state

$$
E=\sum_{\text {bonds }} \frac{1}{2} k_{s}\left(a_{i}-A_{0}\right)^{2}+\sum_{\text {angles }} \frac{1}{2} k_{\theta}\left(\theta_{i}-2 \pi / 3\right)^{2},
$$

it follows that $\partial W / \partial a_{i}=0$ and $\partial W / \partial \theta_{i}=0$ at the equilibrium configuration. Therefore such a lattice has zero bending stiffness, which is not realistic for graphene.

As expected, the infinitesimal bending stiffness of planar graphene does not depend on the direction in which the sheet is bent, i.e., planar graphene is isotropic with respect to bending. For bond-order potentials, the bending modulus adopts the particularly simple form

$$
\mathrm{C}_{\mathrm{b}}=\frac{1}{2} V_{A}\left(A_{0}\right) \bar{B}^{\prime}(2 \pi / 3,2 \pi / 3) \text {. }
$$

\section{Comparison to atomistic calculations}

The explicit expressions for Young's modulus, Poisson's ratio, the shear modulus, and the bending modulus derived in the previous sections are here checked against atomistic calculations. The second parameter set of the potential proposed by Brenner ${ }^{28}$ has been adopted in the comparisons, but the methodology is in principle applicable to any analytical interatomic potential. According to Eqs. (14), (16), (17), (19), and (23):

$$
Y_{s}=235.8 \mathrm{~J} / \mathrm{m}^{2}, \quad \nu_{s}=0.4123, \quad G_{s}=83.47 \mathrm{~J} / \mathrm{m}^{2},
$$

TABLE I. Elastic properties of graphene from $a b$ initio calculations, and from Brenner's potentials [* from Kudin et al. (2001)].

\begin{tabular}{lccc}
\hline \hline & $Y_{s}\left(\mathrm{~J} / \mathrm{m}^{2}\right)$ & $\nu_{s}$ & $\mathrm{C}_{\mathrm{b}}\left(\mathrm{eV} \mathrm{\AA} \AA^{2} /\right.$ atom $)$ \\
\hline Brenner (1990) & 236 & 0.412 & 2.2 \\
Brenner (2002) & 243 & 0.397 & 1.8 \\
Ab initio* & 345 & 0.149 & 3.9 \\
\hline \hline
\end{tabular}

$$
\mathrm{C}_{\mathrm{b}}=2.177 \mathrm{eV} \mathrm{\AA} \AA^{2} / \text { atom. }
$$

The result for Young's modulus perfectly matches that obtained in Ref. 37 for the same potential. To compare with values of the Young's modulus provided in units of pressure which assume a thickness $t=0.34 \mathrm{~nm}$, simply operate as follows: $694 \mathrm{MPa}=236 \mathrm{~J} / \mathrm{m}^{2} / 0.34 \mathrm{~nm}$. To illustrate how these moduli represent the behavior of nanotubes of finite radius, for a $(10,10)$ nanotube we obtain $Y_{s}=234 \mathrm{~J} / \mathrm{m}^{2}$ and $\nu_{s}$ $=0.414$.

To extract Young's modulus and Poisson's ratio of planar graphene from atomistic calculations, a planar slab of graphene is subject to a small uniaxial deformation $(0.02 \%)$. The energy of the structure is then minimized while allowing for lateral deformation. Appropriate periodic boundary conditions are used. The axial force and the lateral deformation are measured from the calculations to compute the elastic moduli. These calculations perfectly match (to four significant digits) the analytical values for $Y_{s}$ and $\nu_{s}$ in Eq. (24).

The significance of the inner displacements on $Y_{s}$ and $\nu_{s}$ can be explored by omitting the second term in the right hand side of Eq. (13), that is omitting the underlined terms of Eqs. (16) and (17). In this case, $Y_{s}^{\text {no inner }}=337.8 \mathrm{~J} / \mathrm{m}^{2}$ and $\nu_{s}^{\text {no inner }}=0.1580$. Atomistic calculations in which the structure is stretched without relaxation of the inner displacements also give these values of elastic moduli. These values differ substantially from those in Eq. (24), so the effect of the inner displacements is very significant. It can be observed that the effect of the inner displacements is very important. Although these elastic moduli agree more closely with accurate $a b$ initio data (see Table I), they do not represent the actual behavior of atomistic systems described by Brenner's potential.

To validate the expression for the bending modulus, Eq. (23), we compute the strain energies (relative to the ground state of planar graphene) of fully relaxed nanotubes of varying radii. We plot the strain energy of these nanotubes versus the inverse of their equilibrium radius, and compare with the simple quadratic expression in the curvature $1 / 2 \mathrm{C}_{\mathrm{b}}(1 / R)^{2}$, where $\mathrm{C}_{\mathrm{b}}$ is not fitted, but obtained from Eq. (23), and $R$ denotes the radius of the nanotube. One would expect this approximation to be valid for very small curvatures, that is very large nanotubes. However, a quadratic approximation to the bending energy has been shown to accurately describe $a b$ initio results for a wide range of radii. ${ }^{16,17}$ Figure 3 shows that the agreement between the atomistic calculations and the quadratic approximation of the energy is excellent. For small nanotubes (right side of the plot) some deviations are noticeable. Nevertheless, for nanotubes of diameter larger than 1 


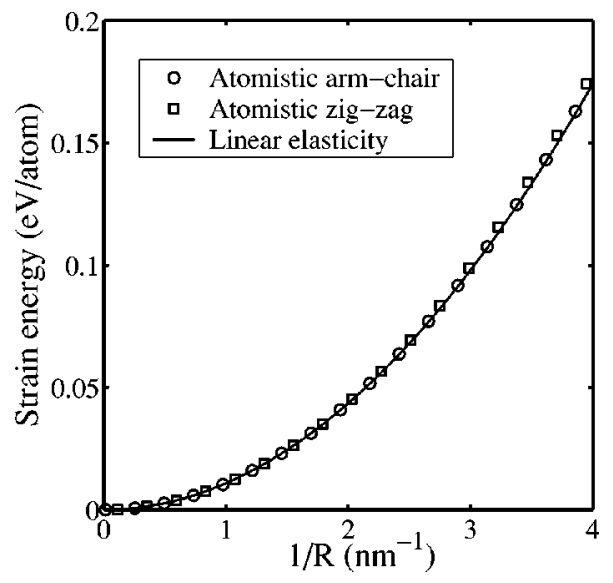

FIG. 3. Strain energy relative to planar graphene for fully relaxed nanotubes of varying radius plotted vs a quadratic approximation of the bending energy with the bending modulus predicted by the continuum theory.

$\mathrm{nm}$, the bending modulus provided by the continuum analysis very accurately characterizes the elasticity of atomistic models of nanotubes.

Table I reports the values of the elastic moduli of planar graphene $Y_{s}, \nu_{s}$, and $\mathrm{C}_{\mathrm{b}}$ derived from Eqs. (14), (16), (17), (19), and (23) for the second parameter set of Brenner's potential. The predicted equilibrium bond length for graphene with this potential is $0.145 \mathrm{~nm}$. The recently developed second generation bond-order potential ${ }^{29}$ is also analyzed, and the associated elastic properties reported in Table I. The equilibrium length predicted by this potential is closer to the widely accepted value of $0.142 \mathrm{~nm}$. The overestimation of the equilibrium bond length with the original potential by Brenner slightly biases the elastic properties. These moduli are compared with available ab initio calculations, ${ }^{17}$ which agree very well with other published data. ${ }^{16}$ It can be observed that the bond-order potentials deviate significantly from the ab initio data. The second generation bond-order potential slightly improves Young's modulus and Poisson's ratio, but provides a worse bending stiffness.

Equation (23) provides valuable insight into the relation between the elastic bending modulus and the functional form of the potential. Consider the following exercise. Let us rescale the bond-order potential, leaving $\bar{B}(2 \pi / 3,2 \pi / 3)$ unchanged but changing $\bar{B}^{\prime}(2 \pi / 3,2 \pi / 3)$ to fit the ab initio value for $C_{b}$. Note that this does not alter the ground energy of graphene, nor the equilibrium bond length. By doing this, we obtain a value for Young's modulus of $336 \mathrm{~J} / \mathrm{m}^{2}$, very close to the $a b$ initio value. Poisson's ratio also dramatically improves to a value of 0.16 . This exercise illustrates how the expressions provided in the previous sections facilitate fitting an analytical potential.

\section{FINITE ELEMENT SIMULATIONS}

The finite deformation continuum theory for a surface without thickness described in Sec. III is now combined with the finite element (FE) method. Since the continuum energy depends on second derivatives of the deformation map through the curvature tensor, a sufficiently smooth finite element approximation is required; here, subdivision finite elements are used. ${ }^{56}$ An alternative coarse grained simulation method for carbon nanotubes based on constrained atomistic calculations in combination with meshfree methods, in the spirit of the nonlocal quasicontinuum method, has been proposed recently. ${ }^{57}$

The continuum/FE approach is validated by comparing the finite element calculations with zero temperature atomistic calculations. The second parameter set of the Brenner potential $^{28}$ is used for the bonded interactions. Since the Brenner potential does not include nonbonded interactions, these are incorporated by a separate potential. ${ }^{58} \mathrm{~A}$ LennardJones potential is adopted for the nonbonded interactions, which corresponds to the graphene-graphene parameter set in Ref. 59. This potential for the nonbonded interactions has been used for instance to analyze the mechanics of $\mathrm{C}_{60}$ molecules inside carbon nanotubes. ${ }^{60}$ Since we want to model intratube van der Waals interactions, in the simulations a given atom can interact via the nonbonded potential with all other atoms in the system, except the set of atoms close to it in the lattice (within the cutoff radius of the nonbonded potential in the undeformed configuration). The main disadvantage of such a partitioning approach, namely that it hinders the reactive capability of the bond-order potential, is irrelevant for our purposes. A continuum version of the nonbonded interactions can also be formulated, ${ }^{13}$ and is implemented in the FE simulations. This continuum nonbonded energy, together with the internal energy in Eq. (10), define the total energy of the continuum system. In both atomistic and continuum simulations, the total energy is minimized with the BFGS quasi-Newton method, which only requires gradients of the energy. The inner relaxation of Eq. (8) is performed numerically by Newton's method at each quadrature point of the finite element model. These bivariate minimization problems typically converge to machine precision in two iterations.

Figure 4 shows the comparisons for a twisted $(10,10)$ nanotube $25 \mathrm{~nm}$ long. In this example, the atomistic system has 12000 degrees of freedom, while the finite element model around 10000 . This example is not intended to demonstrate the computational savings that the continuum/finite element approach can deliver; its purpose is to illustrate the accuracy of the continuum theory in an example involving severe deformations and structural instabilities.

The twisting angle, defined as the total relative rotation of one end with respect to the other, is incrementally increased, and the total energy is minimized at each step. Three representative snapshots of the deformation process are shown in Fig. 4(a), where the deformed configurations of the atomistic and the continuum simulations are presented together. These snapshots demonstrate that the morphological agreement between the continuum and atomistic simulations is excellent, and the deformed continuum surface nearly coincides with the positions of the nuclei provided by the atomistic calculation despite the severe deformations.

The evolution of the binding energy is presented for the two models in Fig. 4(b). This example exhibits two structural instabilities. In the first one, a nonuniform deformation mode 


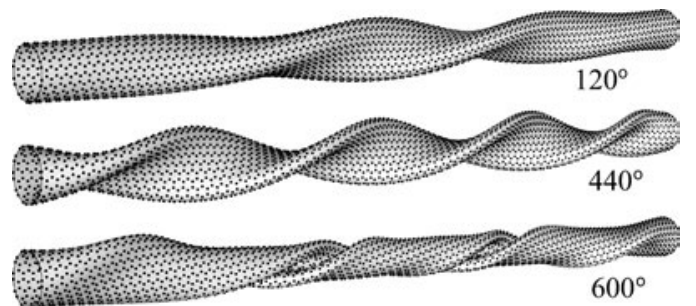

(a)

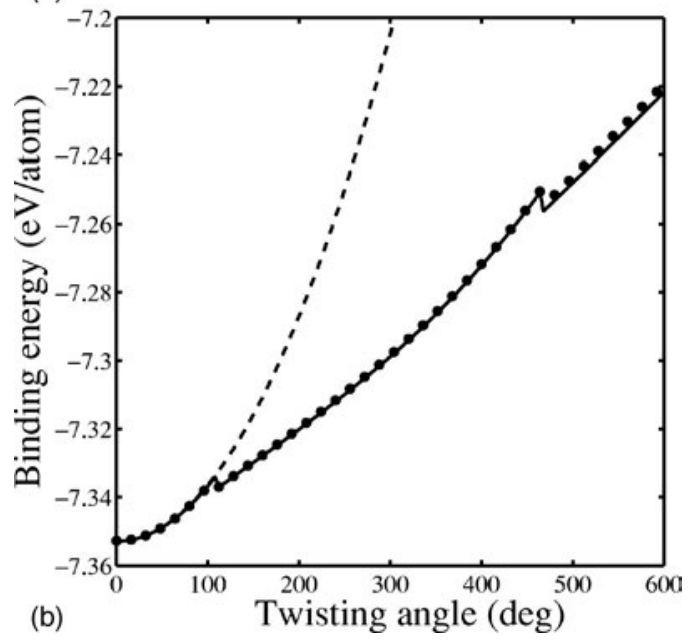

FIG. 4. Twisted 25.1-nm-long (10,10) nanotube: (a) Superimposed deformed configurations at three twisting angles for atomistic calculation (black spheres) and continuum finite element calculation (gray surface). (b) Comparison of the strain energy as a function of the twisting angle for atomistic calculation (-), and continuum/FE calculation ( ), and quadratic approximation with the shear modulus obtained from the continuum analysis (- - -).

develops for a twisting angle of about $100^{\circ}$. The onset of this instability is evident in the first snapshot of the deformation, and can be identified in the strain energy evolution as the kink that ends the nearly quadratic regime. As loading proceeds, the wall of the tube comes into van der Waals contact with itself. Then, the van der Waals interactions slightly stiffen the twisting response of the tube. In their absence, the energy growth after the first instability is roughly linear. The second kink in the strain energy evolution, near $460^{\circ}$, indicates the development of a secondary structure. After this point, the flattened twisted ribbon folds onto itself. Figure 4(b) shows that the energetics of the atomistic system are well predicted by the continuum simulations. In the quadratic regime both methods provide indistinguishable energies. Before the secondary structure develops, at $460^{\circ}$, the discrepancy relative to the total energy change is below $0.4 \%$, and in the final configuration, it is around 3\%. This discrepancy is reduced by refining the finite element mesh. ${ }^{61}$

Figure 4(b) also shows a quadratic approximation of the energy of the twisted nanotube based on linear theory, with the shear modulus analytically derived in Eq. (16). For a homogeneously twisted nanotube, the shear strain of the wall can be written as $\gamma=\Theta R / L$ where $\Theta$ is the twisting angle, $R$ the nanotube radius, and $L$ its length. It can be observed that the quadratic approximation $1 / 2 G_{s} \gamma^{2}$ agrees remarkably

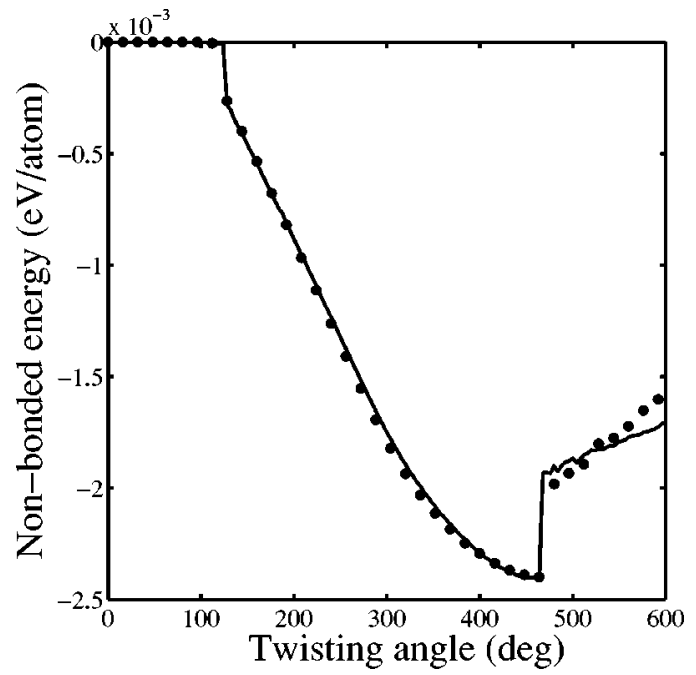

FIG. 5. Twisted 25.1-nm-long $(10,10)$ nanotube: Comparison of the nonbonded energy as a function of the twisting angle for atomistic calculation (-), and continuum/FE calculation $(\mathbf{O})$.

well with the energy of the atomistic system before buckling occurs at $\gamma=5.3 \%$.

Figure 5 shows the evolution of the nonbonded energy, and compares the atomistic and the finite element calculations. The change of nonbonded energy is less than $2 \%$ of the total energy change. Nevertheless, the nonbonded interactions determine the morphology of the deformation, and interpenetration of the wall of the two nanotubes will occur in their absence. Before the first buckle, the nonbonded energy is zero. After the first instability, the system gains van der Waals energy due to the adhesion of the wall that comes into contact with itself. As deformation proceeds, the van der Waals interactions become increasingly repulsive, particularly after the second instability. In this regime, the discrepancies of the finite element prediction for the nonbonded interactions become noticeable. However, this discrepancy in the nonbonded interactions at $600^{\circ}$ is less than $0.1 \%$ of the total energy change.

\section{SUMMARY AND DISCUSSION}

The exponential Cauchy-Born rule has been applied to carbon nanotubes in combination with realistic bond-order potentials. A priori, the application of continuum mechanics to such small systems appears questionable to say the least. However, the presented nonstandard continuum surface theory has been shown to accurately describe both the linear and the nonlinear mechanical response of atomistic systems. Expressions have been developed for the elastic moduli of planar graphene in terms of the atomistic potential. The expression of the bending modulus developed here cannot be obtained from conventional theories. Comparisons with moduli extracted from atomistic calculations show that the analytical expressions very accurately describe the elastic properties of atomistic systems. Comparisons with $a b$ initio elastic moduli suggest that the transferability of commonly used bond-order potentials for hydrocarbons is limited with regards to the elasticity of graphene, and illustrate how these 
explicit expressions can aid the parametrization of analytical potentials.

The application of the theory at finite strains has been presented, and it has been shown that, when combined with the finite element method, it very accurately mimics the nonlinear mechanics of atomistic calculations. An exhaustive set of validation tests is presented in Ref. 61. This reference also illustrates that for nanotubes of large diameters, major computational savings can be achieved by the continuum calculations. In Ref. 30, thanks to the computational efficiency of the proposed approach, rippling deformations occurring in thick multiwalled nanotubes containing millions of atoms are investigated.

The presented continuum model cannot describe fracture or plasticity; its construction relies on a defect-free lattice. Note however that in the numerical example presented above, these processes can in principle take place in the atomistic model, but do not. This illustrates the severe deformations that CNTs can sustain elastically. Nevertheless, there are situations in which failure cannot be ruled out of the analysis, but still the computational savings afforded by the continuum/FE approach are needed (e.g., the full simulation of experiments of nanotube fracture $\left.{ }^{1}\right)$. In these cases, the continuum model can be coupled with atomistic models. ${ }^{62}$ The continuum model can assess the onset the lattice instability, and thus provide a criterion to switch locally from the continuum to the atomistic model.

\section{ACKNOWLEDGMENT}

We gratefully acknowledge the grant support from the NASA University Research, Engineering and Technology Institute on Bio Inspired Materials (BIMat) under Award No. NCC-1-02037.

\section{APPENDIX A: FORMULA FOR THE EXTENDED KINEMATIC RULE}

The precise definition of the extended kinematic rule is provided operationally in this appendix. See Ref. 13 for a derivation. Let $k_{1}$ and $k_{2}$ denote the principal curvatures of the deformed surface $\Omega$. These can be obtained from the generalized eigenvalue problem

$$
\mathcal{K} \cdot \mathbf{V}=k \mathbf{C} \cdot \mathbf{V} \text {, }
$$

where $\mathbf{C}$ and $\mathcal{K}$ are the Lagrangian expressions of the first and the second fundamental forms of the surface. The associated principal directions pulled-back to the undeformed body, $\mathbf{V}_{1}$ and $\mathbf{V}_{2}$, are normalized with respect to $\mathbf{C}$, so that $\mathbf{V}_{i} \cdot \mathbf{C} \cdot \mathbf{V}_{j}=\delta_{i j}$. The conventional principal directions are tangent to the surface, and can be obtained as $\mathbf{v}_{i}=\mathbf{F} \mathbf{V}_{i}$. We define a local orthonormal basis attached to each point of the surface defined by the unit normal to the surface and the principal directions $\mathbf{v}_{1}$ and $\mathbf{v}_{2}$ (normalized). The components of $\mathbf{w}=\mathbf{F A}$ in this basis are $\left(0, w_{1}, w_{2}\right)$, with $w_{i}=\mathbf{V}_{i} \cdot \mathbf{C} \cdot \mathbf{A}$. By defining $\mathcal{Q}(x)=\sin x / x$, the final expression of the lattice vector $\mathbf{A}$ after deformation in the above defined local basis according to the local approximation of the exponential Cauchy-Born rule is

$$
\mathbf{a}=\left\{\begin{array}{c}
\frac{k_{1}\left(w_{1}\right)^{2}}{2} \mathcal{Q}^{2}\left(\frac{k_{1} w_{1}}{2}\right)+\frac{k_{2}\left(w_{2}\right)^{2}}{2} \mathcal{Q}^{2}\left(\frac{k_{2} w_{2}}{2}\right) \\
w_{1} \mathcal{Q}\left(k_{1} w_{1}\right) \\
w_{2} \mathcal{Q}\left(k_{2} w_{2}\right)
\end{array}\right\} .
$$

The length of a deformed bond is then $a=\|\mathbf{a}\|$ and the angle between two deformed bonds $\mathbf{a}$ and $\mathbf{b}$ can be computed as $\theta=\arccos [\mathbf{a} \cdot \mathbf{b} /(a b)]$. Thus, the bond lengths and angles have been expressed in terms of the continuum strain measures $\mathbf{C}$ and $\mathcal{K}$.

\section{APPENDIX B: DERIVATION OF THE BENDING MODULUS}

\section{Kinematic preliminaries}

To calculate the scalar bending modulus defined in Eq. (20), we consider an initially planar graphene sheet bent about one axis. Thus, Eq. (A2) can be simplified to

$$
\mathbf{a}=\left\{\begin{array}{c}
\frac{\kappa\left(w_{2}\right)^{2}}{2} \mathcal{Q}^{2}\left(\frac{\kappa w_{2}}{2}\right) \\
w_{1} \\
w_{2} \mathcal{Q}\left(\kappa w_{2}\right)
\end{array}\right\},
$$

where $\kappa$ is the nonvanishing principal curvature. Variables evaluated at the ground planar configuration of graphene are denoted by $\left.\right|_{\mathrm{gr}}$. Note that $\left.\mathbf{a}\right|_{\mathrm{gr}}=\left(0, w_{1}, w_{2}\right)$ is parallel to the plane of graphene. In this section, derivatives of lattice geometric quantities with respect to $\kappa$ are computed. These derivatives are evaluated at the planar ground configuration, since the goal is to obtain the infinitesimal bending modulus.

The first derivative of a deformed bond length with respect to $\kappa$ follows $\partial a / \partial \kappa=(1 / a) \mathbf{a} \cdot \partial \mathbf{a} / \partial \kappa$. Note that $\partial \mathbf{a} /\left.\partial \boldsymbol{\kappa}\right|_{\mathrm{gr}}=\left(-\left(w_{2}\right)^{2} / 2,0,0\right)$ is perpendicular to the plane of graphene. Consequently, $\partial a /\left.\partial \kappa\right|_{\mathrm{gr}}=0$.

Analogous arguments lead to $\partial(\cos \theta) /\left.\partial \kappa\right|_{\mathrm{gr}}=0$. Since $\partial \theta / \partial \kappa=(1 / \sin \theta) \partial(\cos \theta) / \partial \kappa$, and for graphene no pair of bonds form an angle of $\pi$ in the ground state, it follows that $\partial \theta /\left.\partial \kappa\right|_{\mathrm{gr}}=0$. One can check that this is not the case for dihedral angles, but we do not consider them here.

A second derivative of $a$ with respect to $\kappa$ and the inner displacements is also needed. It can be computed for each component of the inner displacements $A=1,2$ as

$$
\frac{\partial^{2} a}{\partial \kappa \partial \eta_{A}}=\frac{1}{a}(\underbrace{\underset{\mathbf{a}}{\mathbf{g}}}_{\| \mathbf{g r}} \cdot \underbrace{\frac{\partial^{2} \mathbf{a}}{\partial \kappa \partial \eta_{A}}}_{\perp \text { gr }}+\underbrace{\frac{\partial \mathbf{a}}{\partial \eta_{A}}}_{\| \mathrm{gr}} \cdot \underbrace{\frac{\partial \mathbf{a}}{\partial \kappa}}_{\perp \mathrm{gr}}-\frac{\partial a}{\partial \kappa} \frac{\partial a}{\partial \eta_{A}}) .
$$

The underbraces indicate whether the vectors are parallel or perpendicular to the graphene plane when evaluated at the ground state. Therefore, recalling that $\partial a /\left.\partial \kappa\right|_{\mathrm{gr}}=0$, we conclude that $\partial^{2} a /\left.\partial \kappa \partial \boldsymbol{\eta}\right|_{\mathrm{gr}}=\mathbf{0}$. Analogous calculations show that $\partial^{2} \theta /\left.\partial \kappa \partial \boldsymbol{\eta}\right|_{\mathrm{gr}}=\mathbf{0}$. 
Finally, from Eq. (B1) and the honeycomb geometry of graphene, it follows that

$$
\left.\sum_{i=3}^{3} \frac{\partial^{2} a_{i}}{\partial \kappa^{2}}\right|_{\mathrm{gr}}=\frac{-3}{32} A_{0}^{3},\left.\quad \sum_{i=3}^{3} \frac{\partial^{2} \theta_{i}}{\partial \kappa^{2}}\right|_{\mathrm{gr}}=\frac{-9}{8 \sqrt{3}} A_{0}^{2} .
$$

\section{Bending modulus}

Analogous to Eq. (13), the modulus in Eq. (20) can be computed in terms of $W$ when the inner displacements are relaxed as

$$
\mathrm{C}_{\mathrm{b}}=\frac{\partial^{2} W}{\partial \boldsymbol{\kappa}^{2}}-\frac{\partial^{2} W}{\partial \kappa \partial \boldsymbol{\eta}} \cdot\left(\frac{\partial^{2} W}{\partial \boldsymbol{\eta}^{2}}\right)^{-1} \cdot \frac{\partial^{2} W}{\partial \boldsymbol{\eta} \partial \kappa} .
$$

For compactness, the bonds lengths and angles are denoted by the array $\mathbf{p}=\left[a_{1}, a_{2}, a_{3}, \theta_{1}, \theta_{2}, \theta_{3}\right]$, and $p_{i}$ denotes the $i$ th entry of this array. By the chain rule, the first term on the right hand side of Eq. (B4) can be written as

$$
\frac{\partial^{2} W}{\partial \kappa^{2}}=\sum_{i=1}^{6}\left[\sum_{j=1}^{6} \frac{\partial^{2} W}{\partial p_{i} \partial p_{j}} \frac{\partial p_{i}}{\partial \kappa} \frac{\partial p_{j}}{\partial \kappa}+\frac{\partial W}{\partial p_{i}} \frac{\partial^{2} p_{i}}{\partial \kappa^{2}}\right] .
$$

From the derivations of Appendix B 1, one concludes that the first term on the right hand side of the equation above vanishes at the planar ground configuration.
We can check that the second term on the right hand side of Eq. (B4) also vanishes by expanding

$$
\frac{\partial^{2} W}{\partial \boldsymbol{\eta} \partial \kappa}=\sum_{i=1}^{6}\left[\sum_{j=1}^{6} \frac{\partial^{2} W}{\partial p_{i} \partial p_{j}} \frac{\partial p_{i}}{\partial \boldsymbol{\eta}} \frac{\partial p_{j}}{\partial \kappa}+\frac{\partial W}{\partial p_{i}} \frac{\partial^{2} p_{i}}{\partial \boldsymbol{\eta} \partial \kappa}\right],
$$

and recalling the results of Appendix B 1. Thus, the infinitesimal bending modulus around the planar state can be written as

$$
\mathrm{C}_{\mathrm{b}}=\sum_{i=1}^{6} \frac{\partial W}{\partial p_{i}} \frac{\partial^{2} p_{i}}{\partial \kappa^{2}}
$$

At the ground state of graphene and for a bond-order potential, we have

$$
\frac{\partial W}{\partial a_{i}}=\frac{1}{S_{0}}\left(V_{R}^{\prime}-\bar{B} V_{A}^{\prime}\right)=0, \quad \frac{\partial W}{\partial \theta_{i}}=-\frac{2 \bar{B}^{\prime} V_{A}}{S_{0}} .
$$

Recalling the identities in Eq. (B3), it follows that the infinitesimal bending stiffness of planar graphene is

$$
\mathrm{C}_{\mathrm{b}}=\frac{1}{2} V_{A} \bar{B}^{\prime} .
$$

*Now at the Division of Engineering and Applied Science, California Institute of Technology, Pasadena, CA 91125, USA.

${ }^{1}$ M.F. Yu, O. Lourie, M.J. Dyer, K. Moloni, T.F. Kelly, and R.S. Ruoff, Science 287, 637 (2000).

${ }^{2}$ T. Belytschko, S.P. Xiao, G.C. Schatz, and R.S. Ruoff, Phys. Rev. B 65, 235430 (2002).

${ }^{3}$ T. Dumitrica, T. Belytschko, and B.I. Yakobson, J. Chem. Phys. 118, 9485 (2003).

${ }^{4}$ M.B. Nardelli, B.I. Yakobson, and J. Bernholc, Phys. Rev. Lett. 81, 4656 (1998).

${ }^{5}$ D. Srivastava, M. Menon, and K. Cho, Phys. Rev. Lett. 83, 2973 (1999).

${ }^{6}$ B.I. Yakobson, C.J. Brabec, and J. Bernholc, Phys. Rev. Lett. 76, 2511 (1996).

${ }^{7}$ S. Iijima, C.J. Brabec, A. Maiti, and J. Bernholc, J. Chem. Phys. 104, 2089 (1996).

${ }^{8}$ R.S. Ruoff, J. Tersoff, D.C. Lorents, S. Subramoney, and B. Chan, Nature (London) 364, 514 (1993).

${ }^{9}$ N.G. Chopra, L.X. Benedict, V.H. Crespi, M.L. Cohen, S.G. Louie, and A. Zettl, Nature (London) 377, 135 (1995).

${ }^{10}$ M.R. Falvo, G.J. Clary, R.M. Taylor, V. Chi, F.P. Brooks, S. Washburn, and R. Superfine, Nature (London) 389, 582 (1997).

${ }^{11}$ D.A. Walters, L.M. Ericson, M.J. Casavant, J. Liu, D.T. Colbert, K.A. Smith, and R.E. Smalley, Appl. Phys. Lett. 74, 3803 (1999).

${ }^{12}$ P. Poncharal, Z.L. Wang, D. Ugarte, and W.A. de Heer, Science 283, 1513 (1999).

${ }^{13}$ M. Arroyo and T. Belytschko, J. Mech. Phys. Solids 50, 1941 (2002).

${ }^{14}$ J.P. Lu, Phys. Rev. Lett. 79, 1297 (1997).
${ }^{15}$ S. Govindjee and J.L. Sackman, Solid State Commun. 110, 227 (1999).

${ }^{16}$ D. Sánchez-Portal, E. Artacho, J.M. Soler, A. Rubio, and P. Ordejón, Phys. Rev. B 59, 12678 (1999).

${ }^{17}$ K.N. Kudin, G.E. Scuseria, and B.I. Yakobson, Phys. Rev. B 64, 235406 (2001).

${ }^{18}$ C.Q. Ru, Phys. Rev. B 62, 9973 (2000).

${ }^{19}$ C.F. Cornwell and L.T. Willie, Solid State Commun. 101, 555 (1997).

${ }^{20}$ D.H. Robertson, D.W. Brenner, and J.W. Mintmire, Phys. Rev. B 45, 12592 (1992).

${ }^{21}$ V.N. Popov, V.E. Van Doren, and M. Balkanski, Phys. Rev. B 61, 3078 (2000).

${ }^{22}$ E. Hernández, C. Goze, P. Bernier, and A. Rubio, Appl. Phys. A: Mater. Sci. Process. 68, 287 (1999).

${ }^{23}$ T. Xiao and K. Liao, Phys. Rev. B 66, 153407 (2002).

${ }^{24}$ M.F. Yu, T. Kowalewski, and R.S. Ruoff, Phys. Rev. Lett. 86, 87 (2001).

${ }^{25}$ C.Q. Ru, Phys. Rev. B 62, 10405 (2000).

${ }^{26}$ E. Hernández, C. Goze, P. Bernier, and A. Rubio, Phys. Rev. Lett. 80, 4502 (1998).

${ }^{27}$ J. Tersoff, Phys. Rev. B 37, 6991 (1988).

${ }^{28}$ D.W. Brenner, Phys. Rev. B 42, 9458 (1990).

${ }^{29}$ D.W. Brenner, O.A. Shenderova, J.A. Harrison, S.J. Stuart, B. Ni, and S.B. Sinnott, J. Phys.: Condens. Matter 14, 783 (2002).

${ }^{30}$ M. Arroyo and T. Belytschko, Phys. Rev. Lett. 91, 215505 (2003).

${ }^{31}$ M. Born and K. Huang, Dynamical Theory of Crystal Lattices (Oxford University Press, Oxford, 1954).

${ }^{32}$ J.H. Weiner, Statistical Mechanics of Elasticity (Wiley, New York, 1983).

${ }^{33}$ I. Stakgold, Q. Appl. Math. 8, 169 (1950). 
${ }^{34}$ J.W. Martin, J. Phys. C 8, 2837 (1975).

${ }^{35}$ C.S.G. Cousins, J. Phys. C 11, 4867 (1978).

${ }^{36}$ J.L. Ericksen, Phase Transformations and Material Instabilities in Solids, edited by M.E. Gurtin (Academic Press, New York, 1984), pp. 61-77.

${ }^{37}$ P. Zhang, Y. Huang, P.H. Geubelle, P.A. Klein, and K.C. Hwang, Int. J. Solids Struct. 39, 3893 (2002).

${ }^{38}$ R. Al-Jishi and G. Dresselhaus, Phys. Rev. B 26, 4514 (1982).

${ }^{39}$ R. Hill, Math. Proc. Cambridge Philos. Soc. 77, 225 (1975).

${ }^{40}$ F. Milstein, in Mechanics of Solids, edited by H.G. Hopkins and M.J. Sewell (Pergamon, Oxford, 1982).

${ }^{41}$ E.B. Tadmor, M. Ortiz, and R. Phillips, Philos. Mag. A 73, 1529 (1996).

${ }^{42}$ E.B. Tadmor, G.S. Smith, N. Bernstein, and E. Kaxiras, Phys. Rev. B 59, 235 (1999).

${ }^{43}$ G.S. Smith, E.B. Tadmor, and E. Kaxiras, Phys. Rev. Lett. 84, 1260 (2000).

${ }^{44}$ E.B. Tadmor, U.V. Waghmare, G.S. Smith, and E. Kaxiras, Acta Mater. 50, 2989 (2002).

${ }^{45}$ L.E. Malvern, Introduction to the Mechanics of a Continuous Medium (Prentice-Hall, Englewood Cliffs, NJ, 1969).

${ }^{46}$ J.L. Ericksen, Int. J. Solids Struct. 6, 951 (1970).

${ }^{47}$ G. Friesecke and F. Theil, J. Nonlinear Sci. 12, 445 (2002).

${ }^{48}$ J.E. Marsden and T.J.R. Hughes, Mathematical Foundations of Elasticity (Prentice-Hall, Englewood Cliffs, NJ, 1983).

${ }^{49}$ M.P. do Carmo, Differential Geometry of Curves and Surfaces
(Prentice-Hall, Englewood Cliffs, NJ, 1976).

${ }^{50}$ M. Arroyo and T. Belytschko, Mech. Mater. 35, 193 (2003).

${ }^{51}$ U. Dierkes, S. Hildebrandt, A. Küster, and O. Wohlrab, Minimal Surfaces I, Vol. 295 of Comprehensive Studies in Mathematics (Springer-Verlag, Berlin, 1992).

${ }^{52}$ A. Garg, J. Han, and S.B. Sinnott, Phys. Rev. Lett. 81, 2260 (1998).

${ }^{53}$ G.G. Samsonidze, G.G. Samsonidze, and B.I. Yakobson, Phys. Rev. Lett. 88, 065501 (2002).

${ }^{54}$ C. Wei, K. Cho, and D. Srivastava, Phys. Rev. B 67, 115407 (2003).

${ }^{55}$ M. Arroyo, Ph.D. thesis, Northwestern University, 2003.

${ }^{56}$ F. Cirak, M. Ortiz, and P. Schröder, Int. J. Numer. Methods Eng. 47, 2039 (2000).

${ }^{57}$ D. Qian, G.J. Wagner, W.K. Liu, M.F. Yu, and R.S. Ruoff, Appl. Mech. Rev. 55, 495 (2002).

${ }^{58}$ D.W. Brenner, J.A. Harrison, C.T. White, and R.J. Colton, Thin Solid Films 206, 220 (1991).

${ }^{59}$ L.A. Girifalco, M. Hodak, and R.S. Lee, Phys. Rev. B 62, 13104 (2000).

${ }^{60}$ D. Qian, W.K. Liu, and R.S. Ruoff, J. Phys. Chem. B 105, 10753 (2001).

${ }^{61}$ M. Arroyo and T. Belytschko, Int. J. Numer. Methods Eng. 59, 419 (2004).

${ }^{62}$ T. Belytschko and S.P. Xiao, Int. J. Multiscale Comput. Eng. 1, 115 (2003). 